Article

\title{
Clinical Evaluation of INNO-LiPA HPV Genotyping EXTRA II Assay Using the VALGENT Framework
}

\author{
Lan $\mathrm{Xu}^{1,+}{ }^{1}$, Elizaveta Padalko ${ }^{2,+}$, Anja Oštrbenk ${ }^{3}$, Mario Poljak ${ }^{3}\left(\mathbb{D}\right.$ and Marc Arbyn ${ }^{1, *}$ \\ 1 Unit of Cancer Epidemiology, Belgian Cancer Centre, Sciensano (Previously Scientific Institute of Public \\ Health), 1050 Brussels, Belgium; lan.xu@sciensano.be \\ 2 Department of Clinical Chemistry, Microbiology and Immunology, Ghent University Hospital, \\ 9000 Gent, Belgium; elizaveta.padalko@uzgent.be \\ 3 Institute of Microbiology and Immunology, Faculty of Medicine, University of Ljubljana, \\ 1000 Ljubljana, Slovenia; Anja.Ostrbenk@mf.uni-lj.si (A.O.); Mario.Poljak@mf.uni-lj.si (M.P.) \\ * Correspondence: marc.arbyn@sciensano.be; Tel.: +32-2-624-5021 \\ + These authors contributed equally to this work.
}

Received: 12 August 2018; Accepted: 6 September 2018; Published: 11 September 2018

\begin{abstract}
In this diagnostic test validation study, we assessed the clinical accuracy and HPV genotyping performance of the INNO-LiPA HPV Genotyping Extra II (INNO-LiPA) within the VALGENT-3 framework. VALGENT is designed to assess the analytical and clinical performance of HPV tests with genotyping capacity. The VALGENT-3 panel comprised 1300 consecutive cervical cell specimens enriched with 300 samples with abnormal cytology obtained from women attending the Slovenian cervical cancer screening programme. The INNO-LiPA allows type-specific detection of 32 HPV types; however, for the clinical accuracy assessment, we considered it as high-risk (hr)HPV positive when at least one of the following HPV types was present: HPV16, HPV18, HPV31, HPV33, HPV35, HPV39, HPV45, HPV51, HPV52, HPV56, HPV58, HPV59, and HPV68. Clinical accuracy for detection of cervical intraepithelial neoplasia grade 2 or worse (CIN2+) was compared between INNO-LiPA and Hybrid Capture 2 (HC2), which is a standard comparator test for HPV tests used in cervical cancer screening. In addition, hrHPV and type-specific detection HPV types were compared between INNO-LiPA and Linear Array HPV Genotyping Test (Linear Array). The prevalence of hrHPV determined by INNO-LiPA was $17.1 \%$ (95\% CI, 15.0-19.2\%) in the screening population. HrHPV testing with INNO-LiPA had a sensitivity for CIN2+ of 96.9\% (95\% CI, 92.1-99.1\%) which was non-inferior to HC2 (relative sensitivity of $1.01 ; 95 \%$ CI, $0.97-1.04 ; p_{\text {n.inf }}=0.0002$ ) and a specificity for $\leq \mathrm{CIN} 1$ of $85.3 \%$ (95\% CI, 83.2-87.3\%) which was inferior to HC2 (relative specificity of 0.95 ; 95\% CI, 0.93-0.97; $\left.p_{\text {n.inf }}=0.9998\right)$. Genotyping agreement between INNO-LiPA and Linear Array was excellent for hrHPV, HPV16, HPV18, HPV35, HPV45, HPV58 and HPV59, but good or fair for other HPV types. To conclude, INNO-LiPA demonstrated non-inferior clinical sensitivity but lower specificity compared to $\mathrm{HC} 2$ in addition to excellent concordance compared to Linear Array for hrHPV and some genotypes.
\end{abstract}

Keywords: INNO-LiPA; VALGENT; HPV genotyping; cervical cancer; human papillomavirus; clinical validation; Hybrid Capture 2; Linear Array

\section{Introduction}

Over 200 human papillomavirus (HPV) types have been identified and classified based on their nucleotide sequences, with new HPV types being characterized at an increasing rate [1]. Among them, 12 high-risk HPV (hrHPV) types (HPV16, HPV18, HPV31, HPV33, HPV35, HPV39, HPV45, HPV51, HPV52, HPV56, HPV58 and HPV59) are causally linked with cervical cancer and their immediate 
precursors [2]. In addition, eight more HPV types have been associated with some rare cases of cervical cancer (HPV26, HPV53, HPV66, HPV67, HPV68, HPV70, HPV73 and HPV82) [3]. The recognition of the strong etiological association between persistent hrHPV infection and cervical cancer has led to the development of novel HPV tests to enhance secondary prevention of the cervical cancer $[4,5]$. Furthermore, randomized controlled trials (RCTs) have demonstrated that HPV-based screening is more effective than cervical cytology in reducing the incidence of invasive cervical carcinoma in primary screening for cervical cancer for women aged 30 years or older $[5,6]$. Thus, a number of countries are currently in the process of switching from cervical cytology to HPV based primary screening for cervical cancer [7].

Many HPV tests are available on the market but only few have been clinically validated for use in primary screening settings [8]. The Hybrid Capture 2 assay (HC2; Qiagen, Hilden, Germany) [6,9-11] and GP5+/6+ PCR-based enzyme immunoassay (GP5+/6+-EIA; Diassay, Rijwijk, the Netherlands) [12-14] are HPV DNA assays that had been clinically validated for primary screening based on longitudinal evidence obtained from large RCTs. Therefore, HC2 and GP5+/6+-EIA are accepted as the standard comparator tests in evaluations of alternative HPV tests [15]. Several other HPV tests have been fully or partially validated and demonstrate non-inferior clinical sensitivity and specificity for detection of cervical intraepithelial neoplasia grade 2 or worse (CIN2+) compared to the standard comparator tests and high inter-and intra-laboratory reproducibility $[15,16]$. Majority of validated HPV tests target 13 or $14 \mathrm{hrHPV}$ types in aggregate, but some have limited (partial genotyping for HPV16 and HPV18 only), extended (separate genotyping of HPV16, HPV18 and other hrHPV types) and full (type-specific genotyping of all included types) genotyping ability [16]. Since HPV16 and HPV18 are responsible for approximately $70 \%$ of cervical cancer, partial HPV genotyping for these two types is frequently used in the triage of HPV-positive women $[17,18]$. Although the usefulness of full genotyping of hrHPV types is not yet established, a recent study showed that, in addition to HPV16, HPV31 and HPV33 are more carcinogenic than other hrHPV types, suggesting that wider genotyping may also be clinically valuable [19].

INNO-LiPA HPV genotyping assay, based on the principle of reverse hybridization after highly sensitive PCR amplification with SPF10 primers, have been used for HPV genotyping over two decades [20,21]. During this timeframe, the original assay has undergone several modifications, resulting in a few different versions. The INNO-LiPA HPV Genotyping Extra II assay (INNO-LiPA; Fujirebio Europe, Ghent, Belgium) evaluated in the current study is the most recent assay launched by the company in 2015, targeting 32 types, four types more than the previous version. This new version contains genotype specific probes for more decisive genotyping results, an upgraded SPF10 primer set resulting in improved sensitivity (comparable for all hrHPV types), improved human DNA control primers and provides a ready-to-use amplification reagent [22].

In the present study, the VALidation of HPV GENotyping Tests (VALGENT) framework was used to evaluate the clinical accuracy of INNO-LiPA in comparison with HC2. For the first time, it was verified whether hrHPV testing with INNO-LiPA fulfills the minimal requirements for use in primary cervical cancer screening [15]. In addition, type-specific concordance was compared between the INNO-LiPA and the Linear Array HPV Genotyping Test (Linear Array; Roche Molecular Diagnostics, Branchburg, NJ, USA). HrHPV testing with the latter test was recently clinically validated through the VALGENT network as well and has been proposed as a standard analytical HPV genotyping comparator test to resolve discordant typing results of clinically validated HPV assays [23].

\section{Results}

The characteristics of the VALGENT-3 study population, including demographics, cytological and histological results have been described previously [23,24]. Of the 1600 samples analyzed by INNO-LiPA, four samples showed no signal for human HLA-DPB1 gene control line. These four samples were considered as invalid and therefore excluded for further analysis. Of the 1296 valid samples obtained from screening population, $17.1 \%$ women $(221 / 1296)$ tested positive for the presence 
of any of the $13 \mathrm{hrHPV}$ types by INNO-LiPA. The overall and type-specific prevalence of $13 \mathrm{hrHPV}$ types in the total study population determined by INNO-LiPA is summarized in Table 1. The hrHPV prevalence was $15.2 \%$ in women with NILM (negative for intraepithelial lesion or malignancy) and increased to $42.8 \%, 69.0 \%$ and $86.0 \%$ in women diagnosed with ASC-US (atypical squamous cells of undetermined significance), LSIL (low-grade squamous intraepithelial lesion) and HSIL(high-grade squamous intraepithelial), respectively. The risk ratio (RR) of HSIL compared to women with NILM was highest (RR > 8.00) in women infected with in HPV16, HPV33, HPV18 and HPV45.

Table 1. Overall prevalence of hrHPV (aggregate of 13 types) and of individual hrHPV types detected by INNO-LiPA in the total study population according to baseline cytology.

\begin{tabular}{|c|c|c|c|c|c|}
\hline \multirow{2}{*}{ HPV Type } & \multicolumn{4}{|c|}{ HrHPV Prevalence (No. and \%) by Cytology Results } & \multirow{2}{*}{$\begin{array}{c}\text { Ratio Prevalence } \\
\text { HSIL/NILM } \\
\text { HSIL } \\
(N=114)\end{array}$} \\
\hline & \multicolumn{2}{|c|}{$\begin{array}{c}\text { NILM } \\
(N=1234)\end{array}$} & $\begin{array}{l}\text { ASC-US } \\
(N=131)\end{array}$ & $\begin{array}{c}\text { LSIL } \\
(N=113)\end{array}$ & \\
\hline 13 hrHPV * & 187 (15.2\%) & $56(42.8 \%)$ & $78(69.0 \%)$ & $98(86.0 \%)$ & 5.7 \\
\hline HPV16 & $32(2.6 \%)$ & $12(9.2 \%)$ & 27 (23.9\%) & $56(49.1 \%)$ & 18.9 \\
\hline HPV18 & $12(1.0 \%)$ & $4(3.1 \%)$ & $9(8.0 \%)$ & $10(8.8 \%)$ & 8.8 \\
\hline HPV31 & $54(4.4 \%)$ & $22(16.8 \%)$ & $19(16.8)$ & $23(20.2 \%)$ & 4.6 \\
\hline HPV33 & $11(0.9 \%)$ & $5(3.8 \%)$ & $9(8.0 \%)$ & $11(9.7 \%)$ & 10.8 \\
\hline HPV35 & $3(0.2 \%)$ & $1(0.8 \%)$ & $0(0.0 \%)$ & $2(0.9 \%)$ & 4.5 \\
\hline HPV39 & $16(1.3 \%)$ & $1(0.8 \%)$ & $5(4.4 \%)$ & $2(1.8 \%)$ & 1.4 \\
\hline HPV45 & $6(0.5 \%)$ & $5(3.8 \%)$ & $4(3.5 \%)$ & $5(4.4 \%)$ & 8.8 \\
\hline HPV51 & $31(2.5 \%)$ & $4(3.1 \%)$ & $9(8.0 \%)$ & $5(4.4 \%)$ & 1.8 \\
\hline HPV52 & $27(2.2 \%)$ & $10(7.6 \%)$ & $11(9.7 \%)$ & $7(6.1 \%)$ & 2.8 \\
\hline HPV56 & $11(0.9 \%)$ & $2(1.5 \%)$ & $7(6.2 \%)$ & $5(4.4 \%)$ & 4.9 \\
\hline HPV58 & $9(0.7 \%)$ & $3(2.3 \%)$ & $7(6.2 \%)$ & $5(4.4 \%)$ & 6.3 \\
\hline HPV59 & $11(0.9 \%)$ & $3(2.3 \%)$ & $4(3.5 \%)$ & $0(0.0 \%)$ & 0 \\
\hline HPV68 & $16(1.3 \%)$ & $5(3.8 \%)$ & $7(6.2 \%)$ & $5(4.4 \%)$ & 3.4 \\
\hline
\end{tabular}

NILM, negative for intraepithelial lesion or malignancy; ASC-US, atypical squamous cells of undetermined significance; LSIL, low-grade squamous intraepithelial lesion; HSIL, high-grade squamous intraepithelial. * A positive hrHPV result represents detection of at least one of the $13 \mathrm{hrHPV}$ types included in the HC2: HPV16, HPV18, HPV31, HPV33, HPV35, HPV39, HPV45, HPV51, HPV52, HPV56, HPV58, HPV59 and HPV68. Women infected with multiple HPV types were counted only once.

\subsection{Clinical Performance of the INNO-LiPA}

The accuracy data for the INNO-LiPA and HC2 for the outcomes CIN2+, CIN3+ and $\leq$ CIN1 are shown in Table 2 for the total study population and for women aged 30 years or older. When the whole study population was considered, INNO-LiPA detected 123 of 127 CIN2+ cases and 81 of 82 CIN3+ cases, which corresponds to a sensitivity of $96.9 \%$ (95\% CI, 92.1-99.1) and 98.8\% (93.4-100), respectively. The specificity for $\leq$ CIN1 of INNO-LiPA (1034/1212) was 85.3\% (95\% CI, 83.2-87.3). Similar results were obtained for women aged 30 years or older. 
Table 2. Sensitivity of INNO-LiPA and HC2 for detection of CIN2+ and CIN3+ and specificity of both assays for detection of $\leq \mathrm{CIN} 1$. Analysis was performed separately for the total study population and for women $\geq 30$ years old.

\begin{tabular}{|c|c|c|c|c|c|c|}
\hline \multirow{2}{*}{$\begin{array}{l}\text { Assay, Study Population and } \\
\text { Clinical Outcome }\end{array}$} & \multicolumn{3}{|c|}{ Sensitivity } & \multicolumn{3}{|c|}{ Specificity } \\
\hline & $n / N$ & $\%$ & $95 \%$ CI & $n / N$ & $\%$ & $95 \% \mathrm{CI}$ \\
\hline \multicolumn{7}{|c|}{ INNO-LiPA $^{a}$} \\
\hline \multicolumn{7}{|c|}{ Total study population } \\
\hline CIN2+ & $123 / 127$ & 96.9 & $(92.1-99.1)$ & & & \\
\hline CIN3+ & $81 / 82$ & 98.8 & $(93.4-100)$ & & & \\
\hline$\leq \mathrm{CIN} 1$ & & & & $1034 / 1212$ & 85.3 & $(83.2-87.3)$ \\
\hline \multicolumn{7}{|c|}{ Women $>30$ years old } \\
\hline CIN2+ & $95 / 98$ & 96.9 & (91.3-99.4) & & & \\
\hline CIN3+ & $65 / 66$ & 98.5 & $(91.8-100)$ & & & \\
\hline$\leq \mathrm{CIN} 1$ & & & & $887 / 1009$ & 87.9 & $(85.7-89.9)$ \\
\hline \multicolumn{7}{|c|}{ HC2 } \\
\hline \multicolumn{7}{|c|}{ Total study population } \\
\hline CIN2+ & $122 / 127$ & 96.1 & (91.1-98.7) & & & \\
\hline CIN3+ & $80 / 82$ & 97.6 & (91.5-99.7) & & & \\
\hline$\leq \mathrm{CIN} 1$ & & & & $1092 / 1212$ & 90.1 & $(88.3-91.8)$ \\
\hline \multicolumn{7}{|c|}{ Women $>30$ years old } \\
\hline CIN2+ & $94 / 98$ & 95.9 & (89.9-98.9) & & & \\
\hline CIN3+ & $64 / 66$ & 97.0 & (89.5-99.6) & & & \\
\hline$\leq \mathrm{CIN} 1$ & & & & $935 / 1009$ & 92.7 & $(90.9-94.2)$ \\
\hline
\end{tabular}

${ }^{a}$ Positive INNO-LiPA results represents detection of at least one of the following $13 \mathrm{hrHPV}$ types included in the HC2: HPV16, HPV18, HPV31, HPV33, HPV35, HPV39, HPV45, HPV51, HPV52, HPV56, HPV58, HPV59 and HPV68. $n$, number of cases; $N$, total number of cases; $C I$, confidence interval.

The relative sensitivity of INNO-LiPA compared to HC2 was 1.01 (95\% CI, $0.97-1.04 ; p_{\mathrm{mcn}}=0.6547$; $\left.p_{\text {n.inf }}=0.0002\right)$ for CIN2+ and $1.01\left(95 \% \mathrm{CI}, 0.97-1.06 ; p_{\mathrm{mcn}}=0.5637 ; p_{\text {n.inf }}=0.001\right)$ for CIN3+. The relative specificity of INNO-LiPA for $\leq \mathrm{CIN} 1$ was $0.95\left(95 \% \mathrm{CI}, 0.93-0.97 ; p_{\mathrm{mcn}}=0.0000\right.$; $\left.p_{\text {n.inf }}=0.0000\right)$. Similar results were obtained if the analysis was restricted to women $\geq 30$ years (Table 3).

Table 3. Relative sensitivities for detection of CIN2+ and CIN3+ and relative specificity for detection of $\leq \mathrm{CIN} 1$ of INNO-LiPA versus HC2. Analysis was performed separately for the total study population and for women $\geq 30$ years old.

\begin{tabular}{ccccc}
\hline INNO-LiPA vs. HC2 & Relative Sensitivity & Relative Specificity & $p_{\text {mon }} \mathbf{a}$ & $p_{\text {n.inf }}$ \\
\hline Total study population & & & & \\
\hline CIN2+ & $1.01(0.97-1.04)$ & & 0.6547 & 0.0002 \\
CIN3+ & $1.01(0.97-1.06)$ & & 0.5637 & 0.001 \\
$\leq$ CIN1 & & $0.95(0.93-0.97)$ & $<0.001$ & 0.9998 \\
\hline Women > 30 years old & & & \\
\hline CIN2+ & $1.01(0.96-1.06)$ & & 0.6547 & 0.001 \\
CIN3+ & $1.02(0.96-1.07)$ & $0.95(0.93-0.97)$ & 0.5637 & 0.003 \\
$\leq$ CIN1 & & $<0.001$ & 0.999 \\
\hline
\end{tabular}

a $p$ for the McNemar test for a difference between matched proportions and $p_{\mathrm{mcn}}>0.05$ indicates that the sensitivity or specificity of the INNO-LiPA assay are not significantly different from that of the HC2. ${ }^{b} p$ for the test for non-inferiority. A sensitivity threshold of at least $90 \%$ and a specificity threshold of at least $98 \%$ relative to that of the $\mathrm{HC} 2$ were applied in a non-inferiority score test. $p_{\text {n.inf }}<0.05$ means that the sensitivity or specificity of the INNO-LiPa is not significantly lower than that of the HC2. 


\subsection{Genotyping Agreement between INNO-LiPA and Linear Array}

In the total study population, concordance between INNO-LiPA and Linear Array was assessed at type-specific level and overall for $13 \mathrm{hrHPV}$ types (Table 4). Overall concordance of the two assays for $13 \mathrm{hrHPV}$ types was $93.0 \%$ and the corresponding $\mathrm{k}$ value was 0.805 ( $95 \% \mathrm{CI}, 0.757-0.0854)$, indicating excellent agreement between INNO-LiPA and Linear Array. Similarly, the level of agreement was also excellent for detection of HPV16, HPV18, HPV35, HPV45, HPV58 and HPV59. However, for the identification of other individual types, level of agreement ranged from good to poor between the two assays (Table 4). In addition, INNO-LiPA detected more positive cases than Linear Array for all individual types common to both assays.

Table 4. Agreement (concordance and $k$ values) between the INNO-LiPA and the Linear Array for overall hrHPV positivity and for 29 individual HPV types common to both assays in the total study population.

\begin{tabular}{|c|c|c|c|c|c|c|c|}
\hline HPV Type & $\mathrm{I}+/ \mathrm{L}+$ & I+/L- & $\mathrm{I}-/ \mathrm{L}+$ & $\mathrm{I}-/ \mathrm{L}-$ & Concordance & K $(95 \%$ CI $)$ & $p_{\text {men }}{ }^{a}$ \\
\hline 13 hrHPV $^{b}$ & 318 & 103 & 9 & 1166 & $93.0 \%$ & $0.805(0.757-0.854)$ & $<0.001$ \\
\hline HPV16 & 112 & 16 & 2 & 1466 & $99.0 \%$ & $0.920(0.871-0.969)$ & 0.001 \\
\hline HPV18 & 31 & 6 & 3 & 1556 & $99.4 \%$ & $0.870(0.822-0.920)$ & 0.3173 \\
\hline HPV31 & 68 & 50 & 1 & 1417 & $96.8 \%$ & $0.712(0.664-0.759)$ & $<0.001$ \\
\hline HPV33 & 24 & 13 & 1 & 1558 & $99.2 \%$ & $0.770(0.722-0.818)$ & 0.0013 \\
\hline HPV35 & 5 & 0 & 0 & 1591 & $100.0 \%$ & $1.000(0.951-1.049)$ & 1.0000 \\
\hline HPV39 & 15 & 9 & 2 & 1570 & $99.1 \%$ & $0.728(0.680-0.777)$ & 0.0348 \\
\hline HPV45 & 14 & 6 & 0 & 1576 & $99.6 \%$ & $0.822(0.774-0.870)$ & 0.0143 \\
\hline HPV51 & 33 & 16 & 1 & 1546 & $98.9 \%$ & $0.790(0.742-0.838)$ & $<0.001$ \\
\hline HPV52 & 30 & 26 & 2 & 1538 & $94.6 \%$ & $0.674(0.633-0.714)$ & 0.8840 \\
\hline HPV56 & 16 & 9 & 7 & 1564 & $99.0 \%$ & $0.662(0.613-0.712)$ & 0.6171 \\
\hline HPV58 & 19 & 5 & 0 & 1572 & $99.7 \%$ & $0.882(0.833-0.931)$ & 0.0253 \\
\hline HPV59 & 17 & 2 & 2 & 1575 & $99.8 \%$ & $0.894(0.845-0.943)$ & 1.0000 \\
\hline HPV68 & 7 & 26 & 0 & 1563 & $98.4 \%$ & $0.345(0.308-0.382)$ & $<0.001$ \\
\hline HPV26 ${ }^{c}$ & 0 & 0 & 0 & 1596 & $100.0 \%$ & - & 1.0000 \\
\hline HPV53 & 43 & 23 & 1 & 1529 & $98.5 \%$ & $0.774(0.726-0.822)$ & $<0.001$ \\
\hline HPV66 & 29 & 16 & 1 & 1550 & $98.9 \%$ & $0.768(0.720-0.816)$ & $<0.001$ \\
\hline HPV70 & 11 & 10 & 0 & 1575 & $99.4 \%$ & $0.685(0.638-0.731)$ & 0.0016 \\
\hline HPV73 & 19 & 7 & 2 & 1570 & $99.4 \%$ & $0.788(0.739-0.837)$ & 0.0956 \\
\hline HPV82 & 5 & 4 & 1 & 1586 & $99.7 \%$ & $0.665(0.617-0.713)$ & 0.1797 \\
\hline HPV06 & 7 & 14 & 1 & 1574 & $99.1 \%$ & $0.479(0.435-0.523)$ & 0.0008 \\
\hline HPV11 & 2 & 3 & 0 & 1591 & $99.8 \%$ & $0.571(0.526-0.615)$ & 0.0833 \\
\hline HPV40 & 1 & 4 & 0 & 1591 & $99.8 \%$ & $0.333(0.296-0.369)$ & 0.0455 \\
\hline HPV42 & 3 & 5 & 9 & 1579 & $99.1 \%$ & $0.296(0.248-0.344)$ & 0.2850 \\
\hline HPV54 & 10 & 14 & 12 & 1560 & $98.4 \%$ & $0.427(0.378-0.476)$ & 0.6949 \\
\hline HPV61 & 16 & 17 & 6 & 1557 & $98.6 \%$ & $0.575(0.527-0.623)$ & 0.0218 \\
\hline HPV62 & 15 & 12 & 9 & 1560 & $98.7 \%$ & $0.582(0.533-0.632)$ & 0.5127 \\
\hline HPV67 & 3 & 5 & 1 & 1587 & $99.6 \%$ & $0.498(0.452-0.543)$ & 0.1025 \\
\hline HPV81 & 3 & 3 & 1 & 1589 & $99.8 \%$ & $0.599(0.551-0.647)$ & 0.3173 \\
\hline HPV83 & 0 & 6 & 3 & 1587 & $99.4 \%$ & $-0.003(-0.049-0.044)$ & 0.3173 \\
\hline
\end{tabular}

I+ = INNO-LiPA positive; I- = INNO-LiPA negative; L+ = Linear Array positive; L- = Linear Array negative Color legend (adapted from Landis and Koch for the levels of agreement [25]): dark green (1.00 $\geq \kappa>0.80)$ : excellent; light green $(0.80 \geq \kappa>0.60)$ : good; yellow $(0.60 \geq \kappa>0.40)$ : moderate; orange $(0.40 \geq \kappa>0.20)$ : fair; red $(0.20 \geq \kappa>0.00)$ : poor. ${ }^{a} p$ for the McNemar test for a difference between matched proportions and $p_{\text {mcn }}<0.05$ indicates that the HPV positivity detected by INNO-LiPA is significantly different from that of the Linear Array. b 13 hrHPV types: HPV16, HPV18, HPV31, HPV33, HPV35, HPV39, HPV45, HPV51, HPV52, HPV56, HPV58, HPV59 and HPV68. ${ }^{c}$ No HPV26 positive cases detected by both assays, $\mathrm{k}$ value not applicable.

\section{Discussion}

The INNO-LiPA provides full genotyping capability for $32 \mathrm{HPV}$ types. For the purpose of this study, INNO-LiPA was considered positive if at least one of the $13 \mathrm{hrHPV}$ types targeted by HC2 was detected.

To the best of our knowledge, the INNO-LiPA has not been validated previously according to the international guidelines for evaluation of new HPV tests in primary cervical cancer screening settings. Here, we present the first study to evaluate the clinical performance of the INNO-LiPA compared to HC2 using samples from the VALGENT-3 panel. In the whole study population, the INNO-LiPA 
showed a sensitivity for the detection of CIN2+ and CIN3+ of $97 \%$ and $99 \%$, respectively, which was similar to HC2. However, the clinical specificity for $\leq$ CIN1 was only $85 \%$, which was $5 \%(95 \% \mathrm{CI}$, $3-7 \%$ ) lower than the comparator test.

INNO-LiPA is a SPF10 PCR that targets a short highly conserved region in the L1 gene [21,26]. The small size of the amplicon makes the test analytically very sensitive. However, at the same time, discrimination of the individual types is challenging and complex and it is therefore not so surprising that the clinical specificity is lower compared to HPV tests targeting longer DNA sequences [27]. The small size of the amplicon makes INNO-LiPA particularly useful for testing of archived cell preparations or formaline-fixed-paraffin-embedded tissue blocks stored over long periods where parts of the viral genome can be fragmented [28].

INNO-LiPA provides for each HPV type a qualitative output, which is translated into a positive/negative result. The appreciation of presence or absence of blue lines is not quantifiable. Therefore, adaptation of the cut-off, which may allow a more optimal balance between clinical sensitivity and specificity, is in case of INNO-LiPA not possible.

Excellent analytical agreement between INNO-LiPA and Linear Array was observed for $13 \mathrm{hrHPV}$ types overall, HPV16, HPV18, HPV35, HPV45, HPV58 and HPV59. In addition, INNO-LiPA detected more positive cases than Linear Array for all individual HPV types that are common to both assays, with a positivity rate of hrHPV determined by INNO-LiPA statistically significantly higher than that determined by Linear Array $\left(p_{\mathrm{mcn}}<0.001\right)$ in the total study population. The significant difference of positivity rate for hrHPV with these two genotyping methods is consistent with the tests' clinical performances with regard to relative specificity compared to HC2.

In conclusion, in our study, INNO-LiPA exhibits lower clinical specificity; however, this is most likely due to detection of HPV infections with low concentrations and therefore can play an important role in evaluation of viral infection outcomes of vaccination trials and in monitoring the impact of HPV vaccination. Moreover, INNO-LiPA may be useful in epidemiological studies to investigate the prevalence and distribution of HPV types and in studies of the natural history of HPV infection at the type-specific level.

\section{Materials and Methods}

\subsection{Clinical Specimens}

The VALGENT framework is designed to assess the comparative analytical and clinical performance of HPV tests that offer limited to full genotyping capability [29]. VALGENT is iterative, using specimens collected in different countries. The first two VALGENT panels have been completed, using samples collected from Belgium [30-32] and Scotland [33-36]. The third study panel (VALGENT-3) was collated in Slovenia as previously described [11,23,24]. Briefly, 1300 consecutive cervical samples were collected from women who participated in the organised Slovenian national cervical cancer screening program between December 2009 and August 2010 (screening population). The study panel was enriched with 300 cytologically abnormal specimens collected between January 2014 and May 2015 (enrichment population). As required in the VALGENT protocol [29], the enrichment population included 100 women with atypical squamous cervical cells of undetermined significance (ASC-US), 100 women with low-grade squamous intraepithelial lesion (LSIL) and 100 women with high-grade squamous intraepithelial lesion (HSIL).

The sample collection, aliquoting procedure and storage details have been described in detail in previous VALGENT-3 manuscripts [23,24]. Briefly, in July 2016, Ghent University (Ghent Belgium) received 1600 samples of extracted DNA. DNA was extracted from original samples collected into ThinPrep PreservCyt solution (Hologic, Marlborough, MA, USA). Prior to DNA extraction, $1 \mathrm{~mL}$ aliquot of original ThinPrep sample was centrifuged at $13,000 \times g$ for $15 \mathrm{~m}$ with supernatant being discarded and cellular pellet resuspended in $200 \mu \mathrm{L}$ PBS buffer. DNA extraction was performed using QIAamp MinElute Media Kit (Qiagen, Hilden, Germany) following the manufacturer's instructions. 
Bound DNA was finally eluted with $50 \mu \mathrm{L}$ of ATE buffer and stored at $-70{ }^{\circ} \mathrm{C}$ prior to further testing. According to the manufacturer's instructions, we have used $10 \mu \mathrm{L}$ of extracted DNA for INNO-LiPA testing. Similarly, from the second aliquot of original ThinPrep DNA was extracted for Linear Array testing, where $50 \mu \mathrm{L}$ of extracted DNA was used for further testing.

\subsection{HPV Testing}

\subsubsection{INNO-LiPA HPV Genotyping Extra II}

The INNO-LiPA is a line probe assay based on the principle of reverse hybridization for qualitative detection and identification of 32 different HPV types, including 13 hrHPV (HPV16, HPV18, HPV31, HPV33, HPV35, HPV39, HPV45, HPV51, HPV52, HPV56, HPV58, HPV59 and HPV68), 6 possible hrHPV (HPV26, HPV53, HPV66, HPV70, HPV73 and HPV82), 9 low-risk HPV (HPV6, HPV11, HPV40, HPV42, HPV43, HPV44, HPV54, HPV61 and HPV81) plus 4 other HPV genotypes (HPV62, HPV67, HPV83 and HPV89). INNO-LiPA uses the biotinylated consensus primers (SPF10) to amplify a 65-bp region within the L1 region of multiple alpha HPV types. The resulting biotinylated amplicons are then denatured and hybridized with specific oligonucleotide probes. A primer set for the amplification of the human HLA-DPB1 gene is included to monitor sample quality and extraction. The INNO-LiPA assay (sample incubation, stringent wash and color development) was performed fully automated using the AutoBlot $3000 \mathrm{H}$ (Bio-Rad Laboratories Inc., Hercules, CA, USA). Interpretation of the developed strips was done by scanning and automated interpretation using with the LiRAS for LiPA HPV software (Version 3.01, Fujirebio Europe, Ghent, Belgium). The test was performed in accordance with the manufacturer's instructions.

\subsection{2. $\mathrm{HC} 2$}

HC2 detects 13 hrHPV types (HPV16, HPV18, HPV31, HPV33, HPV35, HPV39, HPV45, HPV51, HPV52, HPV56, HPV58, HPV59, and HPV68) and is accepted as a standard comparator test for the clinical validation of hrHPV DNA assays that may be used for primary cervical cancer screening [15]. For the purpose of the present study, hrHPV positivity for INNO-LiPA was defined as the presence of one or more of the $13 \mathrm{hrHPV}$ targeted by HC2.

\subsubsection{Linear Array}

The Linear Array is an HPV test with full genotyping capacity, which detects 37 high- and low-risk HPV types (HPV6, HPV11, HPV16, HPV18, HPV26, HPV31, HPV33, HPV35, HPV39, HPV40, HPV42, HPV44, HPV45, HPV51, HPV52, HPV53, HPV54, HPV56, HPV58, HPV59, HPV61, HPV62, HPV64, HPV66, HPV67, HPV68, HPV69, HPV70, HPV71, HPV72, HPV73, HPV81, HPV82, HPV83, HPV84, HPV89, and IS39) that is frequently used in virological and epidemiological research. In the present study, Linear Array is used as a comparator test to evaluate the analytical genotyping accuracy of the INNO-LiPA.

\subsection{Clinical Outcome and INNO-LiPA Performance Assessment}

As described in previous VALGENT-3 reports [23,24], cytological assessment and referral of patients with abnormal cytology results to colposcopy were done according to the Slovenian national screening guidelines [37], which are in agreement with European guidelines [38]. Colposcopy-directed punch biopsies were obtained from suspicious areas for final histopathological assessment.

Women with histologically confirmed CIN2+ results were considered as diseased subjects. Due to the fact that women with normal cytological results of negative for intraepithelial lesion or malignancy (NILM) were not referred to colposcopy verification in our study, we considered them as subjects without disease only if they had two or more consecutive NILM cytological results (at enrolment and at subsequent screening between 12 to 48 months later). We used this group of women to compute the clinical specificity for $\leq$ CIN1. 
The clinical sensitivity and specificity of the INNO-LiPA for CIN2+ and CIN3+ were calculated. We compared the clinical accuracy of INNO-LiPA to HC2 for CIN2+ and CIN3+, using non-inferiority statistics with a relative sensitivity threshold of $90 \%$ and a relative specificity threshold of $98 \%$ [39]. The McNemar statistic was used in order to compare the differences between matched proportions [40]. For both statistics, the level of significance was set at 0.05 . All analyses were performed using STATA version 14 (Manufacturer, College Station, TX, USA).

Separate and consensus genotyping agreement for the types common to INNO-LiPA and Linear Array was assessed using $\mathrm{K}$ [41] and McNemar statistics [40]. $\mathrm{K}$ values from 0.0 to $0.20,0.21$ to $0.40,0.41$ to $0.60,0.61$ to 0.80 and 0.81 to 1.0 indicate poor, fair, moderate, good and excellent level of agreement between two assays. A McNemar $p$-value of $<0.05$ indicates significant discordance between genotyping results determined by the two assays.

Author Contributions: Conceptualization, M.A.; Data curation, L.X.; Formal analysis, L.X.; Funding acquisition, M.A.; Investigation, E.P.; Methodology, M.A.; Resources, A.O.; Supervision, M.P. and M.A.; Validation, M.P. and M.A.; Writing—original draft, L.X. and E.P.; Writing-review \& editing, A.O., M.P. and M.A.

Funding: L.X., A.O., M.P. and M.A. were supported by the COHEAHR Network (Grant No. 603019), which was funded by the 7th Framework Programme of DG Research and Innovation, European Commission (Brussels, Belgium).

Acknowledgments: Fujirebio Europe provided INNO-LiPA HPV genotyping Extra II kits to the Department of Clinical Chemistry, Microbiology and Immunology, Ghent University Hospital.

Conflicts of Interest: M.A.'s and L.X.'s institution has received support from VALGENT projects, as described previously in the VALGENT protocol paper [29]. M.P.'s and A.O.'s institution received research grants from Abbott Molecular. E.P.'s institution received reagents for testing INNO-LiPA HPV genotyping Extra II assay free of charge from the manufacturer for the duration of the testing.

\section{Abbreviations}

$\begin{array}{ll}\text { INNO-LiPA } & \text { INNO-LiPA HPV Genotyping Extra II assay } \\ \text { HC2 } & \text { Hybrid Capture 2 } \\ \text { Linear Array } & \text { Linear Array HPV Genotyping Test } \\ \text { HPV } & \text { Human papillomavirus } \\ \text { hrHPV } & \text { High-risk HPV } \\ \text { RCT } & \text { Randomised controlled trials } \\ \text { GP5+/6+-EIA } & \text { GP5+/6+ PCR-based enzyme immunoassay } \\ \text { VALGENT } & \text { VALidation of HPV GENotyping Tests } \\ \text { CIN } & \text { Cervical intraepithelial neoplasia } \\ \text { CIN2+ } & \text { CIN grade } 2 \text { or worse } \\ \text { CIN3+ } & \text { CIN grade } 3 \text { or worse } \\ \text { ASC-US } & \text { Atypical squamous cells of undetermined significance } \\ \text { LSIL } & \text { Low-grade squamous intraepithelial lesion } \\ \text { HSIL } & \text { High-grade squamous intraepithelial lesion } \\ \text { NILM } & \text { Negative for intraepithelial lesion or malignance }\end{array}$

\section{References}

1. Bzhalava, D.; Eklund, C.; Dillner, J. International standardization and classification of human papillomavirus types. Virology 2015, 476, 341-344. [CrossRef] [PubMed]

2. Biological Agents: IARC Monographs on the Evaluation of Carcinogenic Risks to Humans. Available online: http:/ / publications.iarc.fr/Book-And-Report-Series/Iarc-Monographs-On-The-Evaluation-OfCarcinogenic-Risks-To-Humans / Biological-Agents-2012 (accessed on 11 September 2018).

3. Arbyn, M.; Tommasino, M.; Depuydt, C.; Dillner, J. Are twenty human papillomavirus types causing cervical cancer? J. Pathol. 2014, 234, 431-435. [CrossRef] [PubMed]

4. Bouvard, V.; Baan, R.; Straif, K.; Grosse, Y.; Secretan, B.; Ghissassi, F.E.; Benbrahim-Tallaa, L.; Guha, N.; Freeman, C.; Galichet, L.; et al. A review of human carcinogens-Part B: Biological agents. Lancet Oncol. 2009, 10, 321-322. [CrossRef] 
5. Arbyn, M.; Ronco, G.; Anttila, A.; Meijer, C.J.L.M.; Poljak, M.; Ogilvie, G.; Koliopoulos, G.; Naucler, P.; Sankaranarayanan, R.; Peto, J. Evidence regarding HPV testing in secondary prevention of cervical cancer. Vaccine 2012, 30, F88-F99. [CrossRef] [PubMed]

6. Ronco, G.; Dillner, J.; Elfstrom, K.M.; Tunesi, S.; Snijders, P.J.; Arbyn, M.; Kitchener, H.; Segnan, N.; Gilham, C.; Giorgi-Rossi, P.; et al. Efficacy of HPV-based screening for prevention of invasive cervical cancer: Follow-up of four European randomised controlled trials. Lancet 2014, 383, 524-532. [CrossRef]

7. Wentzensen, N.; Arbyn, M.; Berkhof, H.; Brower, M.; Canfell, K.; Einstein, M.H.; Early, C.; Monsonega, J.; Franceschi, S. Eurogin 2016 Roadmap: How HPV knowledge is changing screening practice. Int. J. Cancer 2017, 140, 2192-2200. [CrossRef] [PubMed]

8. Poljak, M.; Kocjan, B.J.; Ostrbenk, A.; Seme, K. Commercially available molecular tests for human papillomaviruses (HPV): 2015 update. J. Clin. Virol. 2016, 76, S3-S9. [CrossRef] [PubMed]

9. Sankaranarayanan, R.; Nene, B.M.; Shastri, S.S.; Jayant, K.; Muwonge, R.; Budukh, A.M.; Hingmire, S.; Malvi, S.G.; Thorat, R.; Kothari, A.; et al. HPV screening for cervical cancer in Rural India. N. Engl. J. Med. 2009, 360, 1385-1394. [CrossRef] [PubMed]

10. Kitchener, H.C.; Almonte, M.; Thomson, C.; Wheeler, P.; Sargent, A.; Stoykova, B.; Gilham, C.; Baysson, H.; Roberts, C.; Dowie, R.; et al. HPV testing in combination with liquid-based cytology in primary cervical screening (ARTISTIC): A randomised controlled trial. Lancet Oncol. 2009, 10, 672-682. [CrossRef]

11. Poljak, M.; Ostrbenk, A.; Seme, K.; Ucakar, V.; Hillemanns, P.; Bokal, E.V.; Jancar, N.; klavs, I. Comparison of Clinical and Analytical Performance of the Abbott RealTime High Risk HPV Test to the Performance of Hybrid Capture 2 in Population-Based Cervical Cancer Screening. J. Clin. Microbiol. 2011, 49, 1721-1729. [CrossRef] [PubMed]

12. Hesselink, A.T.; Bulkmans, N.W.; Berkhof, J.; Lorincz, A.T.; Meijer, C.J.L.M.; Snijders, P.J. Cross-sectional comparison of an automated hybrid capture 2 assay and the consensus GP5+/6+ PCR method in a population-based cervical screening program. J. Clin. Microbiol. 2006, 44, 3680-3685. [CrossRef] [PubMed]

13. Naucler, P.; Ryd, W.; Tornberg, S.; Strand, A.; Wadell, G.; Elfgren, K.; Radberg, T.; Strander, B.; Forslund, O.; Hansson, B.G.; et al. Efficacy of HPV DNA testing with cytology triage and/or repeat HPV DNA testing in primary cervical cancer screening. J. Natl. Cancer Inst. 2009, 101, 88-98. [CrossRef] [PubMed]

14. Rijkaart, D.C.; Berkhof, J.; Rozendaal, L.; van kemenade, F.J.; Bulkmans, N.W.J.; Heideman, D.A.M.; Kenter, G.G.; Cuzick, J.; Snijders, P.J.F.; Meijer, C.J.L.M. Human papillomavirus testing for the detection of high-grade cervical intraepithelial neoplasia and cancer: Final results of the POBASCAM randomised controlled trial. Lancet Oncol. 2012, 13, 78-88. [CrossRef]

15. Meijer, C.J.L.M.; Castle, P.E.; Hesselink, A.T.; Franco, E.L.; Ronco, G.; Arbyn, M.; Bosch, F.X.; Cuzick, J.; Dillner, J.; Heideman, D.A. Guidelines for human papillomavirus DNA test requirements for primary cervical cancer screening in women 30 years and older. Int. J. Cancer 2009, 124, 516-520. [CrossRef] [PubMed]

16. Arbyn, M.; Snijders, P.J.; Meijer, C.J.L.M.; Berkhof, H.; Cuschieri, K.; Kocjan, B.J.; Poljak, M. Which high-risk HPV assays fulfil criteria for use in primary cervical cancer screening? Clin. Microbiol. Infect. 2015, 21, 817-826. [CrossRef] [PubMed]

17. Castle, P.E.; Stoler, M.H.; Wright, T.C., Jr.; Sharma, A.; Wright, T.L.; Behrens, C.M. Performance of carcinogenic human papillomavirus (HPV) testing and HPV16 or HPV18 genotyping for cervical cancer screening of women aged 25 years and older: A subanalysis of the ATHENA study. Lancet Oncol. 2011, 12, 880-890. [CrossRef]

18. Monsonego, J.; Cox, J.T.; Behrens, C.; Sandri, M.; Franco, E.L.; Yap, P.S.; Huh, W. Prevalence of high-risk human papilloma virus genotypes and associated risk of cervical precancerous lesions in a large U.S. screening population: Data from the ATHENA trial. Gynecol. Oncol. 2015, 137, 47-54. [CrossRef] [PubMed]

19. Cuzick, J.; Wheeler, C. Need for expanded HPV genotyping for cervical screening. Papillomavir. Res. 2016, 2, 112-115. [CrossRef] [PubMed]

20. Melchers, W.J.G.; Bakkers, J.M.J.E.; Wang, J.; de Wilde, P.C.M.; Boonstra, H.; Quint, W.G.V.; Hanselaar, A.G.J.M. Technical advance. Short fragment polymerase chain reaction reverse hybridization line probe assay to detect and genotype a broad spectrum of human papillomavirus types. Am. J. Pathol. 1999, 155, 1473-1478. [CrossRef]

21. Kleter, B.; van Doorn, L.J.; Schrauwen, L.; Molijn, A.; Sastrowijoto, S.; ter Schegget, J.; Lindeman, J.; ter Harmsel, B.; Burger, M.; Quint, W. Development and clinical evaluation of a highly sensitive PCR-reverse 
hybridization line probe assay for detection and identification of anogenital human papillomavirus. J. Clin. Microbiol. 1999, 37, 2508-2517. [PubMed]

22. Fujirebio Europe Upgrades its HPV (Human Papillomamvirus) Genotyping Panel with the Launch of the INNO-LiPA HPV Genotyping Extra II Assay. Fujirebio Europe Upgrades its HPV (Human Papillomamvirus). Available online: https://www.fujirebio-europe.com/media-and-events/news/2015/fujirebio-europeupgrades-its-hpv-human-papillomavirus-genotyping-panel (accessed on 11 September 2018).

23. Xu, L.; Ostrbenk, A.; Poljak, M.; Arbyn, M. Assessment of the Roche Linear Array HPV genotyping test within the VALGENT framework. J. Clin. Virol. 2018, 98, 37-42. [CrossRef] [PubMed]

24. Polman, N.J.; Ostrbenk, A.; Xu, L.; Snijders, P.; Meijer, C.J.L.M.; Poljak, M.; Heideman, D.A.M.; Abyn, M. Evaluation of the clinical performance of the HPV-Risk assay using the VALGENT-3 panel. J. Clin. Microbiol. 2017, 55, 3544-3551. [CrossRef] [PubMed]

25. Landis, J.R.; Koch, G.G. The measurement of observer agreement for categorical data. Biometrics 1977, 33, 159-174. [CrossRef] [PubMed]

26. Kleter, B.; van Doorn, L.J.; Schrauwen, L.; van Krimpen, K.; Burger, M.; ter Harmsel, B.; Quint, W. Novel short-fragment PCR assey for highly sensitive broad-spectrum detection of anogenital human papillomaviruses. Am. J. Pathol. 1998, 153, 1731-1739. [CrossRef]

27. Iftner, T.; Villa, L.L. Chapter 12: Human papillomavirus technologies. J. Natl. Cancer Inst. Monogr. 2003, 2003, 80-88. [CrossRef]

28. Arbyn, M.; Andersson, K.; Bergeron, C.; Bogers, J.P.; von Knebel-Doeberitz, M.; Dillner, J. Cervical cytology biobanks as a resource for molecular epidemiology. Methods Mol. Biol. 2011, 675, 279-298. [PubMed]

29. Arbyn, M.; Depuydt, C.; Benoy, I.; Boger, J.; Cuschieri, K.; Schmitt, M.; Pawlita, M.; Geraets, D.; Heard, I.; Gheit, T.; et al. VALGENT: A protocol for clinical validation of human papillomavirus assays. J. Clin. Virol. 2016, 76, S14-S21. [CrossRef] [PubMed]

30. Schmitt, M.; Depuydt, C.; Benoy, I.; Boger, J.; Antoine, J.; Arbyn, M.; Pawlita, M. Prevalence and viral load of 51 genital human papillomavirus types and 3 subtypes. Int. J. Cancer 2013, 132, 2395-2403. [CrossRef] [PubMed]

31. Schmitt, M.; Depuydt, C.E.; Benoy, I.; Bogers, J.; Antoine, J.; Pawlita, M.; Arbyn, M. Viral load of high-risk human papillomaviruses as reliable clinical predictor for the presence of cervical lesions. Cancer Epidemiol. Biomarkers Prev. 2013, 22, 406-414. [CrossRef] [PubMed]

32. Schmitt, M.; Depuydt, C.; Benoy, I.; Boger, J.; Antoine, J.; Arbyn, M.; Pawlita, M. Multiple HPV infections with high viral loads are associated with cervical lesions but do not differentiate grades of cervical abnormalities. J. Clin. Microbiol. 2013, 51, 1458-1464. [CrossRef] [PubMed]

33. Cuschieri, K.; Geraets, D.T.; Moore, C.; Quint, W.; Duvall, E.; Arbyn, M. Clinical and analytical performance of the Onclarity HPV assay using the VALGENT framework. J. Clin. Microbiol. 2015, 53, 3272-3279. [CrossRef] [PubMed]

34. Cuschieri, K.; Geraets, D.; Cuzick, J.; Cadman, L.; Moore, C.; Vanden Broeck, D.; Padalko, E.; Quint, W.; Arbyn, M. Performance of a cartridge based assay for the detection of clinically significant HPV infection—Lessons from VALGENT (Validation of HPV Genotyping Tests). J. Clin. Microbiol. 2016, 54, 2337-2347. [CrossRef] [PubMed]

35. Geraets, D.; Cuschieri, K.; Koning, M.; van Doorn, L.; Snijders, P.; Meijer, C.J.L.M.; Quint, W.; Arbyn, M. Clinical evaluation of a GP5+/6+-based Luminex assay having full high-risk HPV genotyping capability and an internal control. J. Clin. Microbiol. 2014, 52, 3996-4002. [CrossRef] [PubMed]

36. Heard, I.; Cuschieri, K.; Geraets, D.T.; Quint, W.; Arbyn, M. Clinical and analytical performance of the PapilloCheck HPV-Screening assay using the VALGENT framework. J. Clin. Virol. 2016, 81, 6-11. [CrossRef] [PubMed]

37. Smernice za celostno obravnavo žensk s predrakavimi spremembami materničnega vratu. Available online: https://zora.onko-i.si/fileadmin/user_upload/dokumenti/strokovna_priporocila/2011_Smernice_ web.pdf (accessed on 11 September 2018).

38. Arbyn, M.; Anttila, A.; Jordan, J.; Ronco, G.; Schenck, U.; Segnan, N.; Wiener, H.; Herbert, A.L.; von karsa, L. European Guidelines for Quality Assurance in Cervical Cancer Screening. Second Edition-Summary Document. Ann. Oncol. 2010, 21, 448-458. [CrossRef] [PubMed]

39. Tang, N.S.; Tang, M.L.; Chan, I.S. On tests of equivalence via non-unity relative risk for matched-pair design. Stat. Med. 2003, 22, 1217-1233. [CrossRef] [PubMed] 
40. McNemar, Q. Note on the sampling error of the difference between correlated proportions or percentages. Psychometrika 1947, 12, 153-157. [CrossRef] [PubMed]

41. Fleiss, J.L; Levin, B.; Paik, M.C. Statistical Methods for Rates and Proportions, 2nd ed.; John Wiley \& Sons: New York, NY, USA, 2013. 